\title{
Prediction and surveillance of influenza epidemics
}

\author{
Justin R Boyle, Ross S Sparks, Gerben B Keijzers, Julia L Crilly, James F Lind and Louise M Ryan
}

T he 2009 (H1N1) influenza pandemic highlighted the need for forecasting models to assist policy and planning for effective health system responses to epidemics. Adequate forewarning of impending influenza epidemics would allow hospitals to adjust staff rosters, assign dedicated wards for infection control, and establish separate influenza clinics collocated with emergency departments (EDs).

We describe the use of surveillance and forecasting to predict and track influenza outbreaks. Although this work was instigated in response to the 2009 (H1N1) influenza outbreak, the methods apply to future outbreaks of other infectious diseases. There is extensive related research in this area using temporal, spatial, or space-time detection algorithms (eg, regression, smoothing, hidden Markov, and wavelet models), with successful performance reported for both sudden and gradual outbreaks. ${ }^{1-9}$ The objective of much of this modelling is to complement traditional public health monitoring with routine automated data analysis. ${ }^{1}$ Characteristics of outbreaks that have influenced detection include the magnitude of the signal, the shape of the signal, and the timing of the outbreak. $^{2}$ An interesting observation is that, since 2000, prospective health surveillance has moved from weekly or monthly data, to daily and faster data rates. ${ }^{1}$ In general, for detecting changes in recent data behaviour, methods incorporating recent data history have advantages over those producing estimates from longer baselines. ${ }^{3}$

Emergency department data have frequently been used to identify disease patterns, ${ }^{10-15}$ including assessment of the timing and magnitude of daily influenza counts against laboratory confirmed influenza. ${ }^{10}$ We describe three approaches to predicting future epidemics based on emergency department data: surveillance monitoring of influenza presentations using adaptive cumulative sum (CUSUM) plan analysis to signal unusual activity; generating forecasts of expected numbers of presentations for influenza, based on historical data; and using internet search data as outbreak notification among a population. All three are considered applicable to health facilities that routinely collect and code patient arrival data.

\section{ABSTRACT}

Objective: To describe the use of surveillance and forecasting models to predict and track epidemics (and, potentially, pandemics) of influenza.

Methods: We collected 5 years of historical data (2005-2009) on emergency department presentations and hospital admissions for influenza-like illnesses (International Classification of Diseases [ICD-10-AM] coding) from the Emergency Department Information System (EDIS) database of 27 Queensland public hospitals. The historical data were used to generate prediction and surveillance models, which were assessed across the 2009 southern hemisphere influenza season (JuneSeptember) for their potential usefulness in informing response policy. Three models are described: (i) surveillance monitoring of influenza presentations using adaptive cumulative sum (CUSUM) plan analysis to signal unusual activity; (ii) generating forecasts of expected numbers of presentations for influenza, based on historical data; and (iii) using Google search data as outbreak notification among a population.

Results: All hospitals, apart from one, had more than the expected number of presentations for influenza starting in late 2008 and continuing into 2009. (i) The CUSUM plan signalled an unusual outbreak in December 2008, which continued in early 2009 before the winter influenza season commenced. (ii) Predictions based on historical data alone underestimated the actual influenza presentations, with 2009 differing significantly from previous years, but represent a baseline for normal ED influenza presentations. (iii) The correlation coefficients between internet search data for Queensland and statewide ED influenza presentations indicated an increase in correlation since 2006 when weekly influenza search data became available.

Conclusion: This analysis highlights the value of health departments performing surveillance monitoring to forewarn of disease outbreaks. The best system among the three assessed was a combination of routine forecasting methods coupled with an adaptive CUSUM method.

MJA 2011; 194: S28-S33

\section{METHODS}

We collected 5 years of historical data (2005-2009) on presentations and hospital admissions for influenza-like illnesses from the Emergency Department Information System (EDIS) database of 27 Queensland public hospitals. The influenza season assessed each year comprised June, July, August and September. Waiting times and triage categories are routinely reported for these 27 public hospitals.

\section{Influenza-like cases}

Patients with influenza-like symptoms were identified by the following International Classification of Diseases (ICD-10-AM) codes within EDIS:

A08.4 - Viral gastroenteritis

B34.9 - Viral infection

J10.8 - (Influenza old code)

J11.1 - (Influenza new code)
J11.1S - H1N1 influenza (Human swine influenza) suspected

J18.0 - Bronchopneumonia

J18.1 - Lobar pneumonia unspecified

J18.2 - Hypostatic pneumonia unspecified

J18.8 - Pneumonia, atypical

J18.9 - Pneumonia, unspecified

Z04.8 - Medical review

Automatic confirmatory swab analysis for suspected influenza is not routine practice in Queensland EDs; hence, the inclusion of codes for possible cases, based on clinician experience. The specified symptom groups included in the codes listed were chosen in consultation with clinicians working at some of the participating hospitals. Although the codes included cover a broad set of conditions (low specificity), they are adequate to reliably capture the range of diagnoses of patients presenting with influenza.

Admitted patients (patients who required subsequent admission to a hospital bed) 
were defined using EDIS departure status description "admitted" (including to an ED bed), and "admitted to the ED" (ie, to a short-stay or observation ward).

\section{Data analysis}

Data collected were stored and collated using Microsoft Office Excel, version 2007 (Microsoft Corporation, Redmond, Wash, USA). SPSS, version 17.0 (SPSS Inc, Chicago, Ill, USA), was used for descriptive statistics. Analysis of variance (ANOVA) tests were performed using MATLAB, verson 7.2.0.232 (R2006a) (The MathWorks Inc, Natick, Mass, USA) for comparisons of influenza presentations and admissions across multiple years. The level of significance was set at $P<0.05$, with $95 \%$ confidence limits. R, version 2.6.2 2008 (The R Foundation for Statistical Computing), was used for CUSUM plan analysis and additional statistical analysis, and a proprietary algorithm written in Java, version 1.5 (Oracle Corp, Redwood Shores, Calif, USA), and Scala, version 2.8 (EPFL, Lausanne, Switzerland), was used for generating forecasts from historical data.

\section{Models for predicting future epidemics}

\section{Surveillance monitoring}

We used an adaptive CUSUM plan for monitoring non-homogeneous negative binomial counts of ED presentations and hospital admissions for influenza to flag unusual outbreaks. ${ }^{16}$ A transitional negative binomial regression model with a moving window of 730 days was used to construct a 1day-ahead forecast for counts across the influenza seasons of 2005-2009. The regression model included factors such as day of the week, school and public holidays, harmonics to account for seasonal trends, and functions of lag counts to account for changes in infection rates.

This model was used to forecast the expected counts for the next day. The overdispersion parameter for each moving window of data is taken as the 1-day-ahead forecast of over-dispersion. These were used to construct a CUSUM plan for flagging unusual outbreaks that had a false alarm rate of roughly one every 200 days. The CUSUM plan represents the sum of standardised differences between the observed counts and a reference value that is a function of the predicted counts. The reference value can be designed to be efficient at detecting a certain size increase at an early stage.

\section{Historical data forecasts}

Forecasts for ED presentations were generated using historical data. Based on experience, hospital bed managers can identify busy periods (ie, certain days of the week, holiday periods, mass-gathering events) reflecting larger admission numbers. Most ED forecasting models allow for this seasonality by including variables for day of the week, month of the year and holidays, and identifying repeated patterns in the time series data. We have found that days having matching characteristics are considered to be most "similar" to the day of interest in terms of patient demand, and accordingly are expected to provide the best basis for predicting the number of presentations expected on that day.

We extracted all days within the historical data that matched the day type (Sunday, Monday, public holiday, etc) within a 4week window, centred on the day of interest (ie, 2 weeks before and 2 weeks after), for use as the basis for prediction. We then applied a computational predictive model (eg, smoothing) to the extracted data to produce a prediction of patient demand for the specified time period.

\section{Internet search data}

We compared our data with Google's flu trends website (http://www.google.org/ flutrends), which uses aggregated Google search data to estimate influenza "activity" in a selection of countries. Data for Queensland are available for download from 2006 onwards. We also used data downloaded from Google's Insights for Search website (http://www.google.com/insights/search), which allows comparison of search volume patterns. We used the search terms "swine flu" and "flu" to check the consistency of internet search results.
Plots were created using ED presentations and Google Flu Trends data for Queensland, normalised over the winter influenza season. The busiest week across the 4-month period was identified for both Google search data and influenza presentations and assigned a value of 1 . Data in other weeks were then normalised and, for influenza presentations, represented as the proportion of cases and, for Google data, as the proportion of searches, relative to values seen on the busiest days.

Other influenza tracking websites, such as those maintained by the United States Centres for Disease Control and Prevention (http://www.cdc.gov/flu/ and http://healthmap.org/swineflu/) do not provide raw search data for Queensland.

\section{Assessment of prediction models}

The models were assessed during the 2009 southern hemisphere influenza season (June-September) for their potential usefulness in informing response policy.

\section{Ethics approval}

The Queensland Health Central Office Human Research Ethics Committee approved the research.

\section{RESULTS}

\section{Influenza-like cases}

During the 2009 influenza season, there were 380000 ED presentations to Queensland public hospitals, equating to $87 \mathrm{ED}$ presentations to public hospitals for every 1000 people in Queensland. Of these presentations, $9 \%$ were patients with influenzalike symptoms (influenza-like cases). This represented a significant increase over previous years $(P<0.001)$ (Box $1, A)$. Across

1 Presentations (A) and admissions (B) of patients with influenza-like symptoms to public hospitals in Queensland in the influenza seasons of 2005-2009; the 2009 influenza season differed significantly from those of preceding years
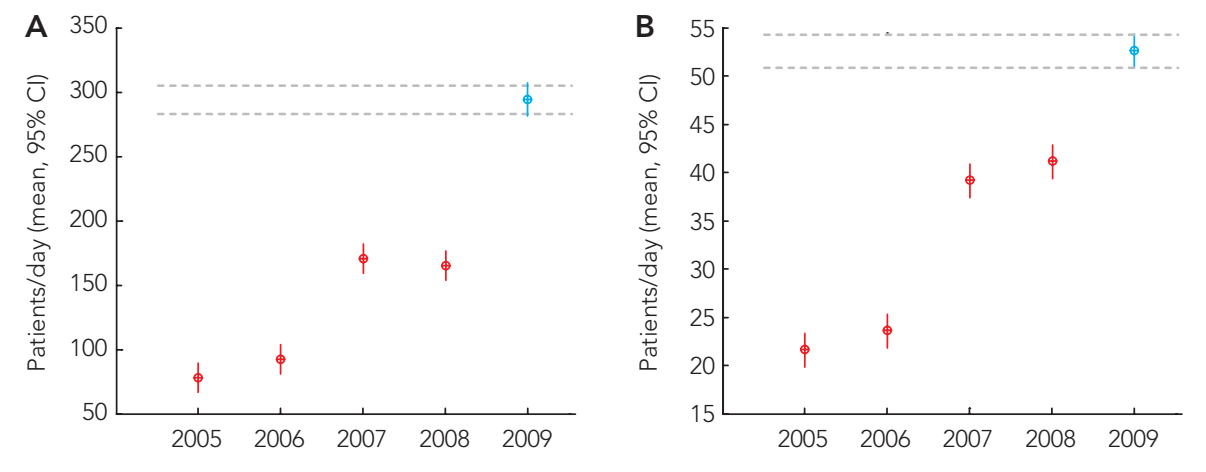


\section{CUSUM plans for signalling unusual presentations and admissions for influenza at three Queensland hospitals}
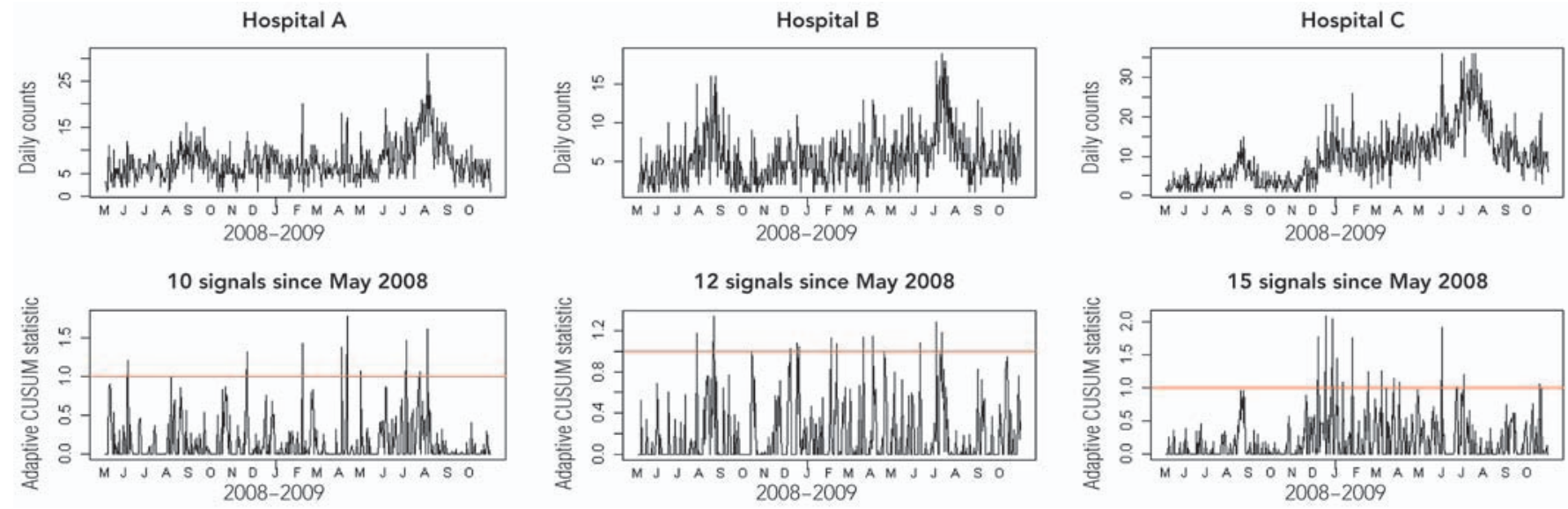

There are 10 non-consecutive signals above the red line, indicating 10 different periods where counts were higher than expected given the influenza counts over the previous 6 days. As we expect only two false alarms for the period, most of these seem to be real alarms, indicating significantly higher than expected counts. It is fairly clear from the control chart above that 2009 was going to be a bad year, with several repeat signals in 2009 prior to the usual winter influenza season.

There are 12 different periods above the red line where counts were higher than expected, indicating late in 2008 and early in 2009 that 2009 was going to be a bad year for influenza. The control chart also identified when the outbreak stopped being unusual, with no alarms in August and September — the more traditional influenza season.
There are 15 non-consecutive signals above the red line, indicating significantly higher than expected counts. Note that the influenza outbreak in 2008 was not higher than expected, but from December 2008 alarms indicated significantly higher than expected counts despite the model adjusting for these. Either the transmission rates were higher than in previous years or the pathogen activity was higher. Again, it is clear that 2009 was going to be a bad year for influenza, and the control chart also identified when the outbreak stopped being unusual. the 2009 winter influenza season, admission rates for patients with and without influenza-like diagnoses were $18 \%$ and $22 \%$, respectively (Box $1, \mathrm{~B}$ ).

\section{Surveillance monitoring}

Examples of surveillance plans for three of the hospitals assessed in our study are shown in Box 2, with the letters (denoting months) on the $x$-axis showing the start of the month. These show a timeseries plot of the counts being monitored (upper panel) and the associated CUSUM plan (lower panel). If the trend in the adaptive CUSUM breaks the red line (the control limit), then an unusual outbreak is signalled, and the CUSUM statistic is reset to zero. A break in the control limit occurring soon after this indicates that the outbreak continues to be significantly worse than expected, despite updating the model using the recent outbreak data.

For example, the CUSUM plan for Hospital $C$ in Box 2 first signals an unusual outbreak in December 2008, but despite the models being updated with this outbreak data, the CUSUM continues to signal that the outbreak remains unusual early in 2009 prior to the commencement of the usual winter influenza season.

\section{Forecasts based on historical data}

Comparing forecasts for ED presentations for influenza-like symptoms with actual presentations in 2009 shows that, at all sites, the historically based forecasts underestimated observed conditions. However, these forecasts represent a baseline for where we would normally expect things to be based on the preceding years. When validating the accuracy of the prediction algorithms across statewide data for the 2009 influenza season, it was found that forecasting accuracy was significantly worse for influenza presentations compared with all ED presentations in 2009, and that the accuracy in July 2009 was significantly worse than that for the surrounding months (Box 3).

\section{Internet search data}

The coherence of Google Trends data with influenza presentation data is shown in Box 4. The correlation coefficients between Google search data for Queensland and statewide ED influenza presentations ( $\rho$ values) indicate an increase in correlation since 2006 when weekly influenza search data became available. While we might expect the peak in influenza ED presentations to lag behind the peak in internet searches related to influenza, the lag was more pronounced in 2009. This may indicate high public and media interest associated with an epidemic.

\section{DISCUSSION}

With routinely collected health data becoming ubiquitous and relatively inexpensive to obtain, the potential for utilising forecasting and surveillance to support the decisions of hospital policymakers is evident.

Our CUSUM analysis identified an unusual influenza outbreak in the Australian summer of 2008-2009, as well as significantly more influenza-like presentations in the following winter. The latter outbreaks coincided with the worldwide H1N1 influenza outbreak. The results of the surveillance monitoring indicated that all hospitals, apart from one, had bigger than expected influenza outbreaks starting in late 2008 and continuing well into 2009. The one hospital that was not as affected by swine influenza was a large children's hospital, which, interestingly, had relatively larger influenza outbreaks in 2007 and 2008 than other hospitals. It could be hypothesised that the high exposure of the source population to the 2007 and 2008 influenza made it less susceptible to influenza in 2009.

Many hospitals have seen the merit of generating forecasts from historical data to manage elective and emergency admissions in a proactive manner. ${ }^{17-22}$ The ability to accurately predict patient flow on a weekly or seasonal basis could enable more 
3 Prospective forecast accuracy for statewide data from 27 Queensland public hospitals, 2005-2009. Upper section (grey): all patients presenting to emergency departments (EDs). Lower section (green): patients with influenza-like diagnoses

All presentations
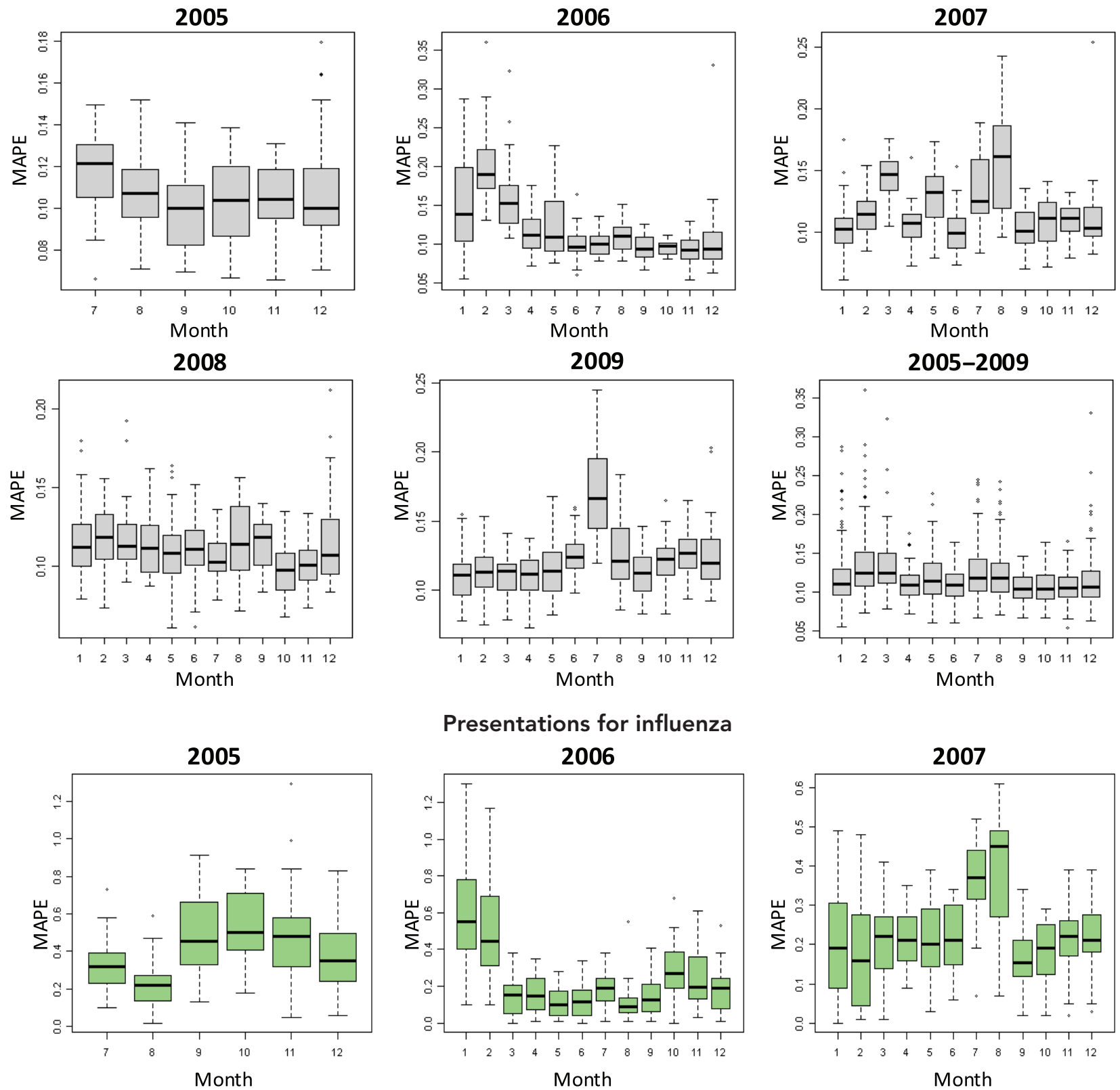

Presentations for influenza
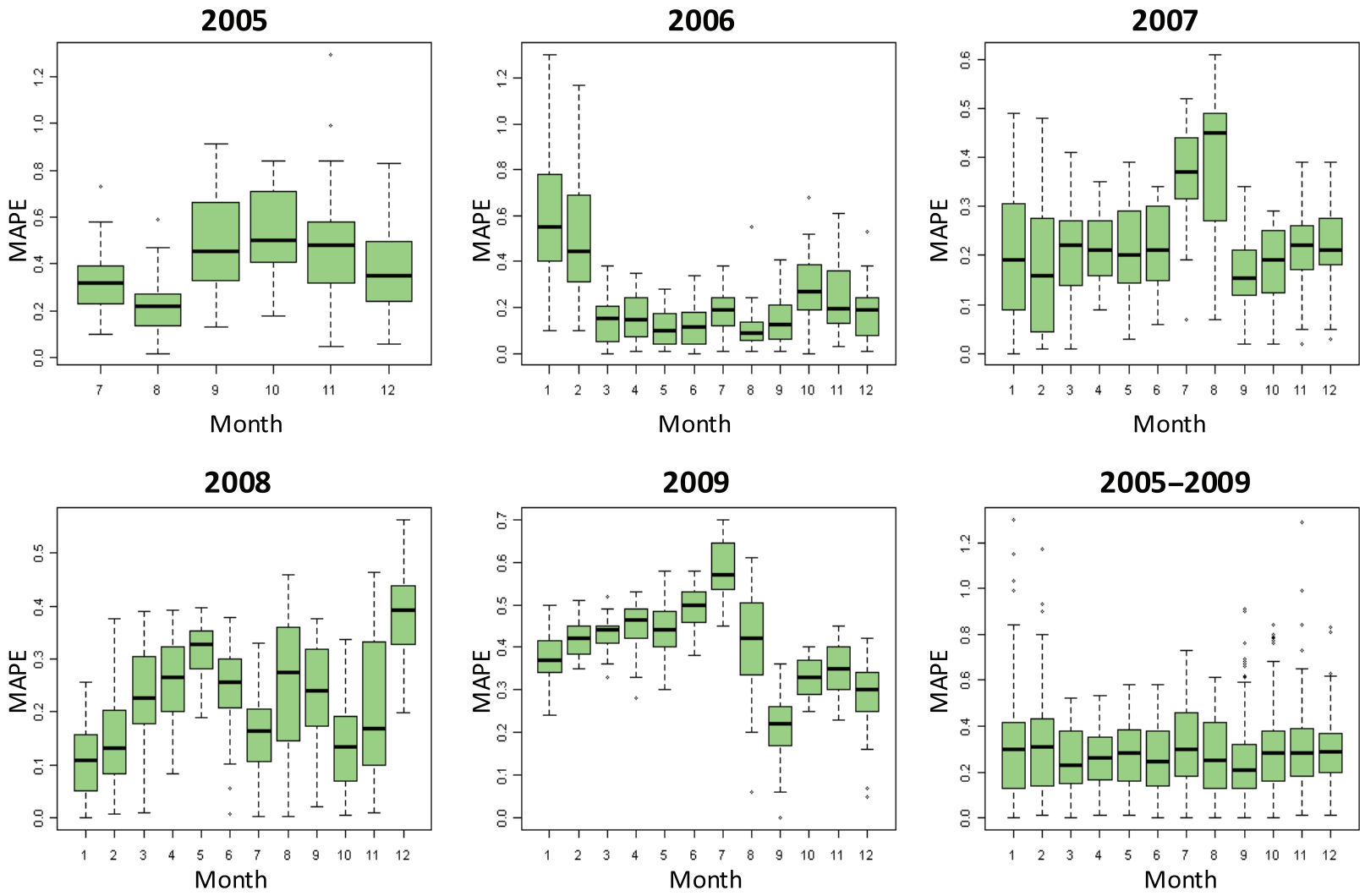


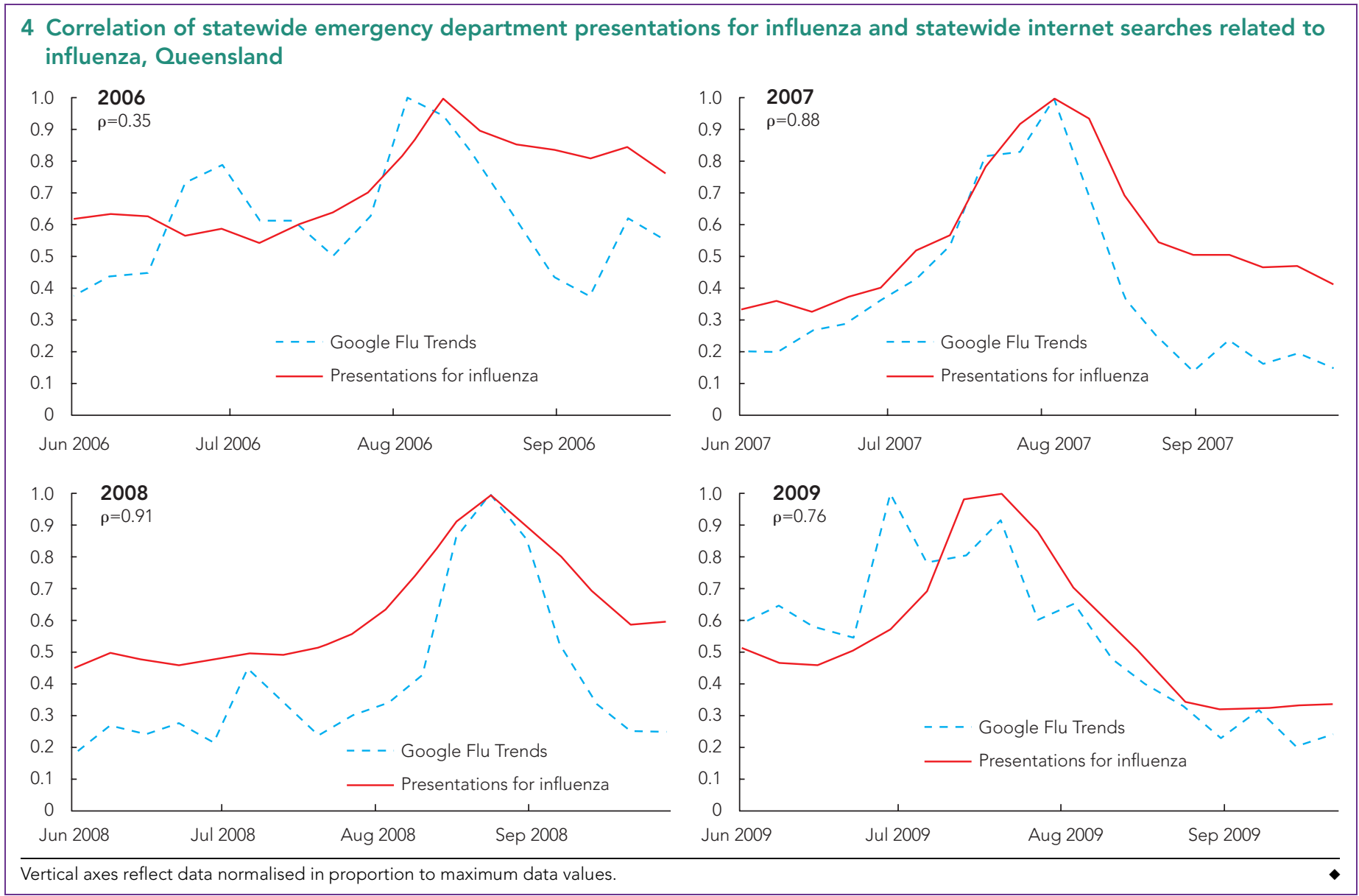

informed decisions about staffing levels to be made, which, in turn, could result in cost savings and improved patient care. Forecasting is a widely applicable, multidisciplinary science, encompassing statisticians, economists and operations researchers, that guides decision making in many areas of economic, industrial and scientific planning, but has gained little traction in the health care industry. ${ }^{22}$ We advocate that all health facilities that routinely collect data relating to workload and bed demand use this information proactively for improving management and patient outcomes. Our analysis shows, however, that routine forecasting methods need to be coupled with an adaptive methodology, such as the CUSUM approach described here, that can detect unusual occurrences.

The value of using internet search data in pandemic planning has been reported previously, ${ }^{23-25}$ with Google publishing correlations between search data and epidemics. ${ }^{23}$ Now these search data are accessible to the lay community and were used to construct the plots in Box 4. Additionally, Google's Insights for Search site can report search interest relating to other infectious diseases to allow state health departments to survey trends for diseases other than influenza. According to Google, raw search volume is not reported, as the size of some regions may skew the data, and normalised data are used instead. The coherence of geographically specific search data with influenza presentations may enable information-poor regions to track the progression of pandemics in the community.

\section{Study limitations}

In the collection of data to build prediction and surveillance models, we have not included data from private hospitals or general practitioners.

Swab analysis was not undertaken to confirm influenza status and is usually only done for certain clinical indications (eg, in patients who are pregnant, patients with comorbidities and those who are immunocompromised). Thus, it is possible that the results do not reflect influenza and may instead relate to other respiratory viruses with characteristics similar to those of influenza. We assumed correct and consistent ICD diagnostic coding, but some cases may not have had influenza-like illnesses. Also, the data are specific to Queensland, which accounts for $20 \%$ of the Australian population. There may be factors relating to larger populations, and different climates and latitudes, which affect the applicability of these results.

There are a number of broad issues associated with how best to achieve accurate surveillance and forecasting. There are many options for the choice of data used (eg, Google influenza searches, ED presentations, hospital admissions, medicine purchases). There are also many different options and approaches to surveillance and forewarning technology, including modelbased approaches, statistical process control methods, and so on. The approach we chose combines model-based prediction with classic process control methods. However, there are a variety of alternatives that could be developed.

A difficulty with the 1-day-ahead surveillance approach is that, if an outbreak is gradual, there is a danger that the models will track the outbreak and thus reduce the differences between observed and forecast values. An alternative could be longer-term 
forecasts, such as 7-day or even longer forecasts. Our CUSUM modelling adapts to local influences by updating the regression parameters on a daily basis by fitting models to a moving window of data. Distinguishing usual from unusual events for the influenza virus is also difficult, because the timing of the seasonal epidemic varies naturally from year to year, as does the impact of the differing virulence of the virus and other factors.

A limitation with the historical data forecasting was the choice of forecast models assessed. Other techniques, such as dynamic regression, state-space models and neural network forecasts were not pursued because of the limited time frame of this study.

Finally, there are several biases in search engine data and caveats in their use. Google searches are less likely to be undertaken by persons under 10 years or over 70 years, although their carers may do so (eg, to clarify symptoms). Also countries with low internet connectivity (eg, regions of Africa and Eastern Europe) will not be represented.

\section{CONCLUSION}

To determine unusual events and predict expected presentation rates, the best system among those assessed was a combination of routine forecasting methods coupled with an adaptive CUSUM methodology. Widely accessible internet search data can also assist by reflecting the timing of epidemics. The use of these forewarning and forecasting models can assist clinicians and hospital managers in clinical and infection control decision making.

\section{ACKNOWLEDGEMENTS}

We would like to acknowledge the support of the Queensland Emergency Medicine Research Foundation, Queensland Health's data custodians, and staff from the Centre for Healthcare Improvement. We thank Mr Remy Le Padellec and Mr Derek Ireland for their contributions to this work. The CSIRO (Commonwealth Scientific and Industrial Research Organisation) holds a patent in the area of forecasting emergency department presentations.

\section{COMPETING INTERESTS}

None identified.

\section{AUTHOR DETAILS}

Justin R Boyle, BEng(Hons), PhD, Research Scientist ${ }^{1}$

Ross S Sparks, MSc, PhD, Research Scientist ${ }^{2}$ Gerben B Keijzers, MB BS, MSc(ClinEpi),

FACEM, Emergency Physician, ${ }^{3}$ and Associate Professor, Bond University

Julia L Crilly, MN(Hons), PhD, Nurse Researcher and Associate Professor ${ }^{4}$

James F Lind, BS BM, BMedSci, FACEM,

Emergency Physician, ${ }^{3}$ and Associate Professor, Griffith University

Louise M Ryan, MA, PhD, Chief ${ }^{2}$

1 CSIRO ICT Centre, Brisbane, QLD.

2 CSIRO Mathematics Informatics and Statistics, Sydney, NSW.

3 Gold Coast Hospital, Southport, QLD. 4 Southern Area Health Service ED Clinical Network, and Griffith University Research Centre for Clinical and Community Practice Innovation, Gold Coast Hospital, Southport, QLD.

Correspondence: justin.boyle@csiro.au

\section{REFERENCES}

1 Burkom HS, Murphy SP, Shmueli G. Automated time series forecasting for biosurveillance. Stat Med 2007; 26: 4202-4218.

2 Buckeridge DL. Outbreak detection through automated surveillance: a review of the determinants of detection. J Biomed Inform 2007; 40: 370-379.

3 Elbert Y, Burkom HS. Development and evaluation of a data-adaptive alerting algorithm for univariate temporal biosurveillance data. Stat Med 2009; 28: 3226-3248.

4 Murphy SP, Burkom H. Recombinant temporal aberration detection algorithms for enhanced biosurveillance. J Am Med Inform Assoc 2008; 15: 77-86.

5 Berger M, Shiau R, Weintraub JM. Review of syndromic surveillance: implications for waterborne disease detection. J Epidemiol Community Health 2006; 60: 543-550.

6 Watkins RE, Eagleson S, Veenendaal B, et al. Disease surveillance using a hidden Markov model. BMC Med Inform Decis Mak 2009; 9: 39.

7 Watkins RE, Eagleson S, Veenendaal B, et al. Applying cusum-based methods for the detection of outbreaks of Ross River virus disease in Western Australia. BMC Med Inform Decis Mak 2008; 8: 37.

8 Fricker RD Jr, Hegler BL, Dunfee DA. Comparing syndromic surveillance detection methods: EARS versus a CUSUM-based methodology. Stat Med 2008; 27: 3407-3429.

9 Cakici B, Hebing K, Grunewald M, et al. CASE: a framework for computer supported outbreak detection. BMC Med Inform Decis Mak 2010; 10: 14

10 Zheng W, Aitken R, Muscatello DJ, et al. Potential for early warning of viral influenza activity in the community by monitoring clinical diag- noses of influenza in hospital emergency departments. BMC Public Health 2007; 7: 250.

11 Schindeler SK, Muscatello DJ, Ferson MJ, et al. Evaluation of alternative respiratory syndromes for specific syndromic surveillance of influenza and respiratory syncytial virus: a time series analysis. BMC Infect Dis 2009; 9: 190.

12 Hope K, Durrheim DN, Muscatello D, et al. Identifying pneumonia outbreaks of public health importance: can emergency department data assist in earlier identification? Aust $N Z J$ Public Health 2008; 32: 361-363.

13 Hope K, Merritt T, Durrheim DN. Evaluating the utility of emergency department syndromic surveillance for a regional public health service. Commun Dis Intell 2010; 34: 310-318.

14 Muscatello DJ, Churches T, Kaldor J, et al. An automated, broad-based, near real-time public health surveillance system using presentations to hospital emergency departments in New South Wales, Australia. BMC Public Health 2005; 5: 141

15 Churches T, Conaty SJ, Gilmour RE, Muscatello $D$. Reflections on public health surveillance of pandemic (H1N1) 2009 influenza in NSW. NSW Public Health Bull 2010; 21(1-2): 19-25.

16 Sparks RS, Keighley T, Muscatello D. Early warning CUSUM plans for surveillance of negative binomial daily disease counts. J Appl Stat 2010; 37: 1911-1929.

17 Hoot N, Aronsky D. An early warning system for overcrowding in the emergency department, AMIA Annu Symp Proc 2006: 339-343.

18 Hoot NR, Zhou C, Jones I, et al. Measuring and forecasting emergency department crowding in real time. Ann Emerg Med 2007; 49: 747-755.

19 McCarthy ML, Zeger SL, Ding R, et al. The challenge of predicting demand for emergency department services. Acad Emerg Med 2008; 15: 337-346.

20 Schweigler LM, Desmond JS, McCarthy ML. Forecasting models of emergency department crowding. Acad Emerg Med 2009; 16: 301-308.

21 Champion R, Kinsman LD, Lee GA, et al. Forecasting emergency department presentations. Aust Health Rev 2007; 31: 83-90.

22 Jones SS, Thomas A, Evans RS, et al. Forecasting daily patient volumes in the emergency department. Acad Emerg Med 2008; 15: 159170.

23 Ginsberg J, Mohebbi MH, Patel RS, et al. Detecting influenza epidemics using search engine query data. Nature 2009; 457: 10121014.

24 Eysenbach G. Infodemiology: tracking flurelated searches on the web for syndromic surveillance. AMIA Annu Symp Proc 2006: 244248.

25 Wilson N, Mason K, Tobias M, et al. Interpreting Google flu trends data for pandemic H1N1 influenza: the New Zealand experience. Euro Surveill 2009; 14 (44). pii: 19386.

(Received 1 Apr 2010, accepted 1 Dec 2010) 